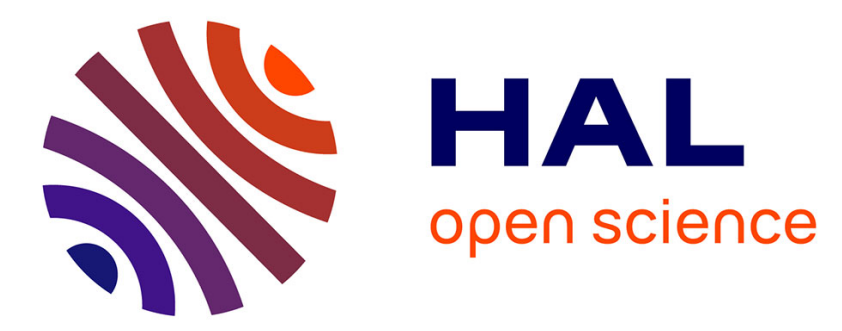

\title{
Simple and distortion-free optical sampling of terahertz pulses via heterodyne detection schemes
}

Jérôme Degert, Marion Cornet, Emmanuel Abraham, Eric Freysz

\section{To cite this version:}

Jérôme Degert, Marion Cornet, Emmanuel Abraham, Eric Freysz. Simple and distortion-free optical sampling of terahertz pulses via heterodyne detection schemes. Journal of the Optical Society of America B, 2016, 33 (10), pp.2045 - 2050. 10.1364/JOSAB.33.002045 . hal-01397369

\section{HAL Id: hal-01397369 \\ https://hal.science/hal-01397369}

Submitted on 15 Nov 2016

HAL is a multi-disciplinary open access archive for the deposit and dissemination of scientific research documents, whether they are published or not. The documents may come from teaching and research institutions in France or abroad, or from public or private research centers.
L'archive ouverte pluridisciplinaire HAL, est destinée au dépôt et à la diffusion de documents scientifiques de niveau recherche, publiés ou non, émanant des établissements d'enseignement et de recherche français ou étrangers, des laboratoires publics ou privés.

\section{다(1)(2)}

Distributed under a Creative Commons Attribution - ShareAlikel 4.0 International 


\title{
Simple and distortion-free optical sampling of terahertz pulses via heterodyne detection schemes
}

\author{
Jérôme Degert, ${ }^{1,2, *}$ Marion Cornet, ${ }^{1,2}$ Emmanuel Abraham, ${ }^{1,2}$ and Eric Freysz ${ }^{1,2}$ \\ 'Univ. Bordeaux, LOMA, UMR 5798, F-33400 Talence, France \\ ${ }^{2}$ CNRS, LOMA, UMR 5798, F-33400 Talence, France \\ ${ }^{*}$ Corresponding author: jerome.degert@u bordeaux.fr
}

\begin{abstract}
We present and demonstrate experimentally two simple and distortion-free methods to sample terahertz pulse in zinc-blende-type crystals like $\mathrm{ZnTe}$ or GaP. They are based on an optical heterodyne detection scheme which makes it possible to measure the terahertz field by detecting the optical probe beam or its second harmonic. Both are compared to conventional electro-optic sampling and give results in good agreement with the latter.
\end{abstract}

OCIS codes: (190.7110) Ultrafast nonlinear optics; (320.7100) Ultrafast measurements; (300.6495) Spectroscopy, terahertz.

\section{INTRODUCTION}

Electro optic (EO) sampling, hereafter reported as conven tional EO sampling (CEOS), is a widely used technique for the measurement of the electric field of a terahertz $(\mathrm{THz})$ pulse with subpicosecond time resolution [1 3]: in a zinc blende crystal, the polarization state of a linearly polarized ultrashort optical pulse is modulated by the $\mathrm{THz}$ pulse through the Pockels effect. The corresponding phase retardation $\Gamma$, propor tional to $\mathrm{THz}$ electric field amplitude $E_{\mathrm{THz}}$, is then measured by ellipsometry for different time delays $\tau$ between the two pulses. The ellipsometer, consisting of a $\lambda / 4$ plate followed by a suitably oriented Wollaston polarizer, converts the phase modulation into an amplitude modulation of the optical probe pulse measured by a balanced photodetector. Different alterna tives to this technique have been proposed, but they suffer from some detection nonlinearities $[4,5]$. Even though these nonli nearities can be corrected, they increase the complexity of the measurement process with respect to conventional EO. Very re cently, new methods based on a technique commonly used in optically heterodyne detected optical Kerr effect spectroscopy have been introduced [6,7]. These distortion free techniques are based on two measurements at opposite optical biases near the zero transmission point in a crossed polarizers detection geometry. They lead to an increase, by 1 order of magnitude, of the signal enhancement factor, defined as the ratio of the normalized detected balanced difference signal to $\Gamma$.

Hereafter, we propose two novel and simple schemes, based on a special geometry of the polarization states of the optical and $\mathrm{THz}$ beams. The first one leads to an amplitude modulation of the optical pulse at the output of the zinc blende crystal and makes it possible to separate the optical pulse generated by the Pockels effect, hereafter called the signal pulse, from the in cident pulse [8]. Thanks to an optical heterodyne detection of these signal pulses, one can perform a distortion free sampling of $\mathrm{THz}$ pulses within a single measurement. In the second one, we take advantage of this geometry where the Pockels nonlinearity couples to the intrinsic second order nonlinearity of the EO crys tal, leading to the generation of a second harmonic signal [the Pockels induced second harmonic (PISH) effect], which can be used, through an optical heterodyne detection, to sample tera hertz pulses [9]. Since the latter detection scheme, which shows some similarities with the first one, offers interesting perspectives for the sampling of $\mathrm{THz}$ pulses generated by near infrared pulses, we will present it here with more details than in [9].

This paper is organized as follows. In Section 2, we present the experimental setup of our optically heterodyne detected EO sampling and derive the expression of the detected signal. The latter expression is then analyzed and confronted with the experiment. Next, in Section 3, we give a comprehensive analysis of the optically heterodyne detected PISH signal and compare it, experimentally, with conventional EO sampling. Finally, in Section 4, we compare both detection schemes.

\section{OPTICALLY HETERODYNE-DETECTED ELECTRO-OPTIC SAMPLING}

\section{A. Experimental Setup}

The experiment is the following [Fig. 1(a)]: a linearly polarized $\mathrm{THz}$ pulse is emitted from air ionized by a two color, namely 
400 and $800 \mathrm{~nm}$, femtosecond laser field [10]. This THz pump field is then collimated and focused onto a $\langle 110\rangle$ EO crystal (ZnTe or GaP), with a thickness $L(L=300 \mu \mathrm{m}$ for $\mathrm{ZnTe}$ and $200 \mu \mathrm{m}$ for $\mathrm{GaP}$ ), by two off axis paraboloidal mirrors with a $150 \mathrm{~mm}$ focal length. Inside the crystal, the $\hat{y}$ polarized $\mathrm{THz}$ beam propagates collinearly with a weak $800 \mathrm{~nm}$ probe beam, initially $\hat{z}$ polarized by a half wave plate and a polarizer, the $\hat{z}$ axis coinciding with the $\langle 001\rangle$ axis of the crystal [Fig. 1(b)]. After the crystal, the transmitted optical probe beam is split by a 50/50 nonpolarizing beam splitter. Both beams are then sent through a quarter wave plate and a polarizer, and their intensity is recorded by photodiodes. The fast axis of the quarter wave plate in front of the photodiode PD1 (resp. PD2) is oriented at an angle $\beta$ (resp. $\beta$ ) with respect to the $\hat{z}$ axis. The axes of the polarizers are set along the $\hat{y}$ axis, so that the incident probe beam is blocked in the absence of $\mathrm{THz}$ and $\beta=0^{\circ}$. However, when the probe and $\mathrm{THz}$ pulses temporally overlap in the crystal, their interactions within the crystal generates a weak optical signal polarized along the $\hat{y}$ axis [8]. The latter is therefore transmitted by the polarizers. The $\mathrm{THz}$ beam is chopped and the signal, $\Delta I(\Gamma, \beta)=I_{1}(\Gamma, \beta) \quad I_{2}(\Gamma, \beta)$, mea sured by the two photodiodes versus the time delay $\tau$ between the probe and $\mathrm{THz}$ pulses, is recorded by a lock in amplifier.

\section{B. Electro-Optic Sampling by Optical Heterodyne Detection}

Let us see what is the expression of the signal $\Delta I(\Gamma, \beta)$ provided by the setup of Fig. 1 . In the $(\hat{\mathbf{y}}, \hat{\mathbf{z}})$ basis, the electric field of the incident probe beam writes

$$
\mathbf{E}=E_{0}\left[\begin{array}{l}
0 \\
1
\end{array}\right]
$$

At the output of the EO crystal, after interaction with the $\mathrm{THz}$ pulse, this field is given by [8]

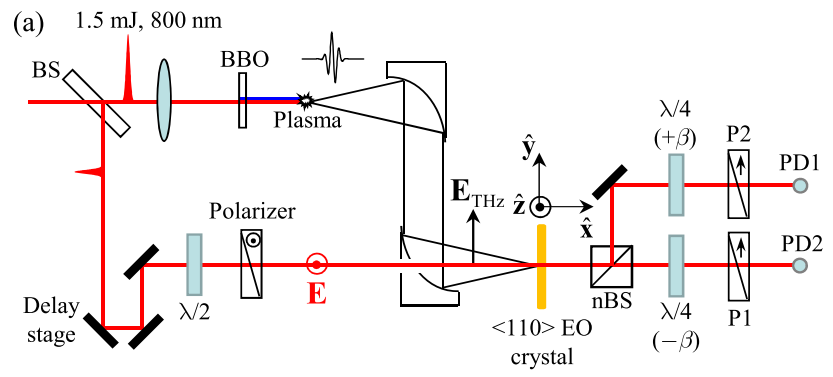

(b)

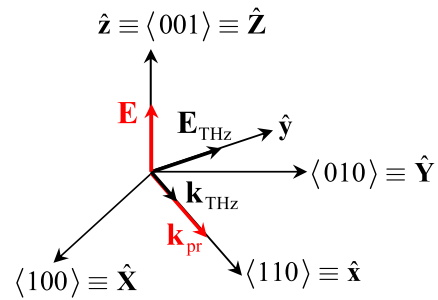

Fig. 1. (a) Experimental setup. BS, beam splitter; nBS, nonpolariz ing beam splitter; P, polarizer; PD, photodiode. (b) Geometry of the experiment. $(\hat{\mathbf{X}}, \hat{\mathbf{Y}}, \hat{\mathbf{Z}})$, Cartesian frame of the crystal; $(\hat{\mathbf{x}}, \hat{\mathbf{y}}, \hat{\mathbf{z}})$, Cartesian frame of the laboratory. $\mathbf{k}_{\mathrm{THz}}$ (resp. $\mathbf{k}_{\mathrm{pr}}$ ) is the wave vector of the $\mathrm{THz}$ (resp. probe) pulse.

$$
\mathbf{E}=E_{0}\left[\begin{array}{c}
\mathrm{i} \sin (\Gamma / 2) \\
\cos (\Gamma / 2)
\end{array}\right]
$$

with

$$
\Gamma=\frac{2 \pi L}{\lambda} n_{\omega}^{3} r_{41} E_{\mathrm{THz}}
$$

the phase retardation induced by the THz field, where $\lambda$ is the wavelength of the probe beam, $n_{\omega}$ is the refractive index of the crystal in the optical range, and $r_{41}$ its electro optic coefficient. Thus, the electric field of the signal pulse is given by $E_{\text {sig }}=$ $\mathrm{i} E_{0} \sin (\Gamma / 2) \simeq \mathrm{i} E_{0} \Gamma / 2$ in the weak $T H z$ field limit $(\Gamma \ll 1)$.

Using the Jones matrix formalism [11], one can derive the expression of the electric fields $E_{1}$ and $E_{1}$ transmitted by polar izers $P_{1}$ and $P_{2}$, respectively, and their intensities $I_{1}$ and $I_{2}$ detected by photodiodes PD1 and PD2, respectively. All cal culations done, one has

$I_{1}(\Gamma, \beta)=\frac{I_{0}}{2}\left[\sin ^{2}\left(\frac{\Gamma}{2}\right) \cos ^{2}(2 \beta)+\frac{1}{2} \sin ^{2}(2 \beta)+\frac{1}{2} \sin (2 \beta) \sin \Gamma\right]$

and $I_{2}(\Gamma, \beta)=I_{1}(\Gamma, \beta)$. The second term in Eq. (4) corre sponds to the intensity of the local oscillator,

$$
I_{\mathrm{LO}}=I_{1}(0, \beta)=\frac{I_{0}}{4} \sin ^{2}(2 \beta),
$$

and the intensity of the homodyne signal on PD1, $I_{\text {sig }}$, is given by

$$
I_{\text {sig }}=I_{1}(\Gamma, 0)=\frac{I_{0}}{2} \sin ^{2}\left(\frac{\Gamma}{2}\right) .
$$

Now, if one considers the modulation depth $M(\Gamma, \beta)=$ $\left[I_{1}(\Gamma, \beta) \quad I_{1}(0, \beta)\right] / I_{1}(0, \beta)$ of the signal detected by PD1 [6], it is then possible to express the enhancement factor $g$ and the distortion parameter $d$ associated with our method. They are related to the modulation depth, in the weak field limit, by

$$
M(\Gamma, \beta)=g \Gamma+d(g \Gamma)^{2}+O\left(\Gamma^{3}\right) .
$$

From Eqs. (4) and (5), one has

$$
\begin{gathered}
g=\frac{1}{\sin (2 \beta)}, \\
d=\frac{1}{2} \cos ^{2}(2 \beta) .
\end{gathered}
$$

Equation (8) agrees with the expression of the enhancement factor found for EO sampling expressed in the terminology of optical heterodyne detection [6], where the heterodyning of the signal is performed in front of the EO crystal. On the other hand, the distortion parameter we found is multiplied by a fac tor $\cos ^{2}(2 \beta)$ compared to [6], meaning that our detection scheme is less sensitive to distortion as $\beta$ increases. However, since the enhancement factor is better for small values of $\beta, d \simeq$ $1 / 2$ over the optimal operating range of our detection scheme.

Finally, this distortion is removed by measuring the subtrac tion of the signals detected by both photodiodes,

$$
\Delta I(\Gamma, \beta)=\frac{I_{0}}{2} \sin (2 \beta) \sin \Gamma,
$$

whose enhancement factor is $2 g$. 


\section{Results and Discussion}

Figure 2 displays the $\mathrm{THz}$ electric fields and their spectra, de tected with our setup when ZnTe [Figs. 2(a) and 2(b)] or GaP [Figs. 2(c) and 2(d)] crystals are used, for values of the angle $\beta$ ranging from $5^{\circ}$ to $29^{\circ}$. For comparison, all these fields are nor malized to one and superimposed to the normalized $\mathrm{THz}$ fields measured with a CEOS setup, as described in [1], using the same EO crystal. At first sight, there is a very good agreement between both detection schemes, especially in terms of signal to noise ratio, which is about $10^{3}$. Discrepancies between the signals, if any, might be due to some small mismatch between the heterodyning angles of the quarter wave plates, leading to a nonvanishing contribution from the homodyne signal, which would distort the measured signal shape. We kept such a source of distortion as small as possible by tuning the angle of the quarter wave plates so that the intensities of the local oscillator on each photodiode, $I_{1}(0, \beta)$ and $I_{2}(0, \beta)$, respectively, were equal, the signals delivered by PD1 and PD2 being measured by means of a voltmeter.

However, a closer look at the detected waveforms, especially their dependence with respect to the heterodyning angle $\beta$ [Figs. 3(a) and 3(c)], reveals some discrepancies. This is clearly evidenced by Figs. 3(b) and 3(c), which display the evolution of the peak value of the nonnormalized signal $\Delta I$ with respect to the angle $\beta$. According to Eq. (10), this amplitude is expected to scale like $\sin (2 \beta)$. For $\beta \leq 20^{\circ}$, the experimental results are in agreement with this angular dependence, as shown by the fits on Figs. 3(b) and 3(d). But, for higher values of $\beta$, the peak value of $\Delta I$ saturates and no longer obeys to Eq. (10). We attribute this behavior, and the subsequent distortion of the detected $\mathrm{THz}$ waveforms, to the saturation of PD1 and PD2 by the local oscillator signal, $I_{\mathrm{LO}}$, once $\beta>20^{\circ}$. Nevertheless, this saturation of the photodetectors is not detrimental since, according to Eq. (8), it is better to work at small values of $\beta$.
Indeed, the smaller the heterodyning angle $\beta$, the better is the enhancement factor $2 g$ of EO sampling by optical heterodyne detection. For instance, for $\beta=5^{\circ}$, the enhancement factor is $2 g \simeq 1 / \beta \sim 10$, a value identical to the enhancement factor obtained in [7].

\section{TERAHERTZ PULSES SAMPLING THROUGH OPTICALLY HETERODYNE-DETECTED PISH SIGNAL}

In a recent paper [9], we have shown that it was possible to detect $\mathrm{THz}$ pulses via the detection of the second harmonic (SH) of an optical pulse induced through its interaction with an intense $\mathrm{THz}$ pulse in a zinc blende crystal (the so called PISH effect). Briefly, with the geometry of Fig. 1, an optical signal pulse is induced by the $\mathrm{THz}$ pulse through the Pockels effect. For the sake of simplicity, assuming monochro matic plane waves at angular frequencies $\omega$ in the optical range and $\Omega$ in the $\mathrm{THz}$ range, the electric field of the signal is $E_{\text {sig }}=E_{y}^{(\omega \pm \Omega)} \simeq E_{y}^{\omega}$, since $\Omega \ll \omega$. This field then couples to the fundamental electric field, $E_{z}^{\omega}$, through the second order nonlinearity of the crystal, $\chi_{X Y Z}^{(2)}$, to generate a second harmonic signal with an electric field given by [9]: $E_{\mathrm{PISH}} \propto$ $\chi_{X Y Z{ }^{(2)} r_{1}}\left(E_{z}^{\omega}\right)^{2} E_{\mathrm{THz}} L / \lambda$. Since the PISH signal is proportional to the $\mathrm{THz}$ electric field, it has been used to detect the latter. However, such a detection requires to heterodyne the PISH signal in order to avoid losing the phase of the $\mathrm{THz}$ waveform. This was done by inserting a quarter wave plate on the optical beam pathway in front of the crystal.

Recently, to circumvent some limitations of lithium niobate or $\mathrm{ZnTe}$ (two or three photon absorption in the spectral range of the pump, absorption in the $\mathrm{THz}$ range, phase mismatch), new materials have been developed to generate intense $\mathrm{THz}$ pulses by optical rectification: orientation patterned GaAs [12] or stilbazolium salt single crystals [13], for example.
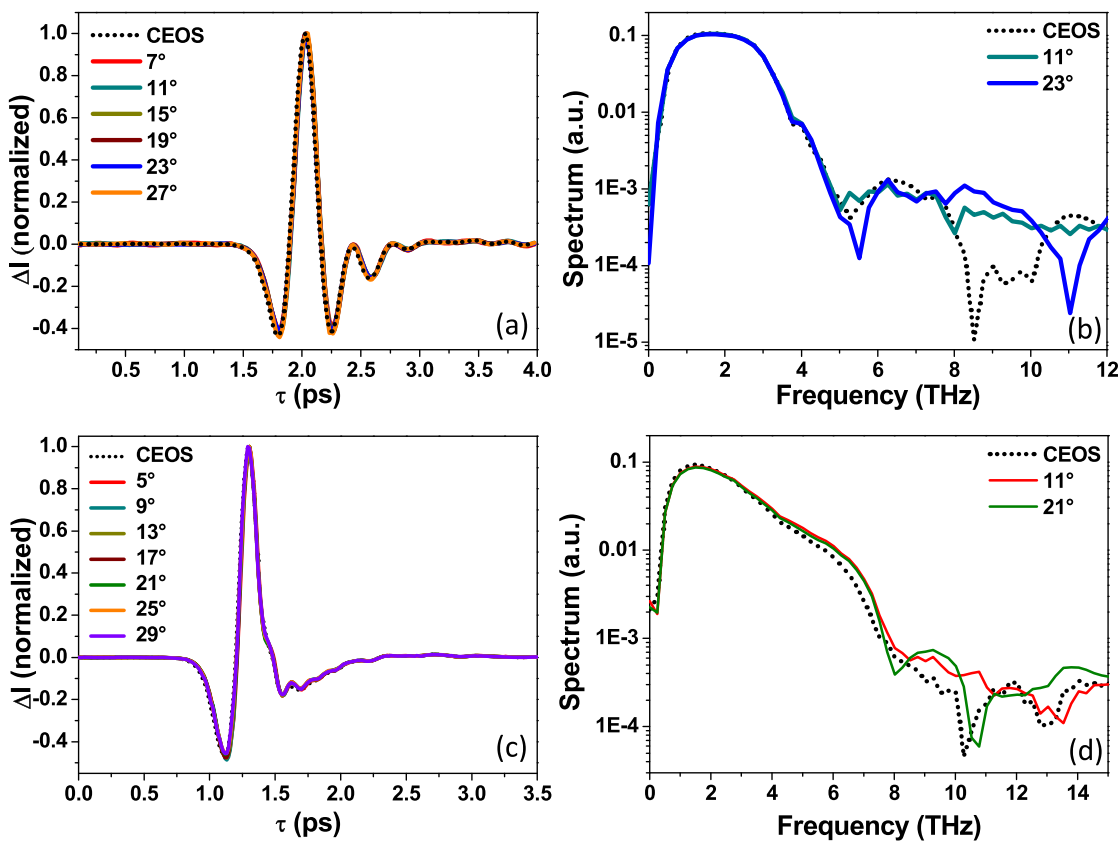

Fig. 2. EO sampling by optical heterodyne detection performed in ZnTe [(a) and (b)] and GaP [(c) and (d)]. (a),(c) Normalized THz electric field, for different $\beta$ angles, versus the time delay $\tau$ compared to the field measured with a CEOS setup (dotted line). (c),(d) Corresponding spectra. 

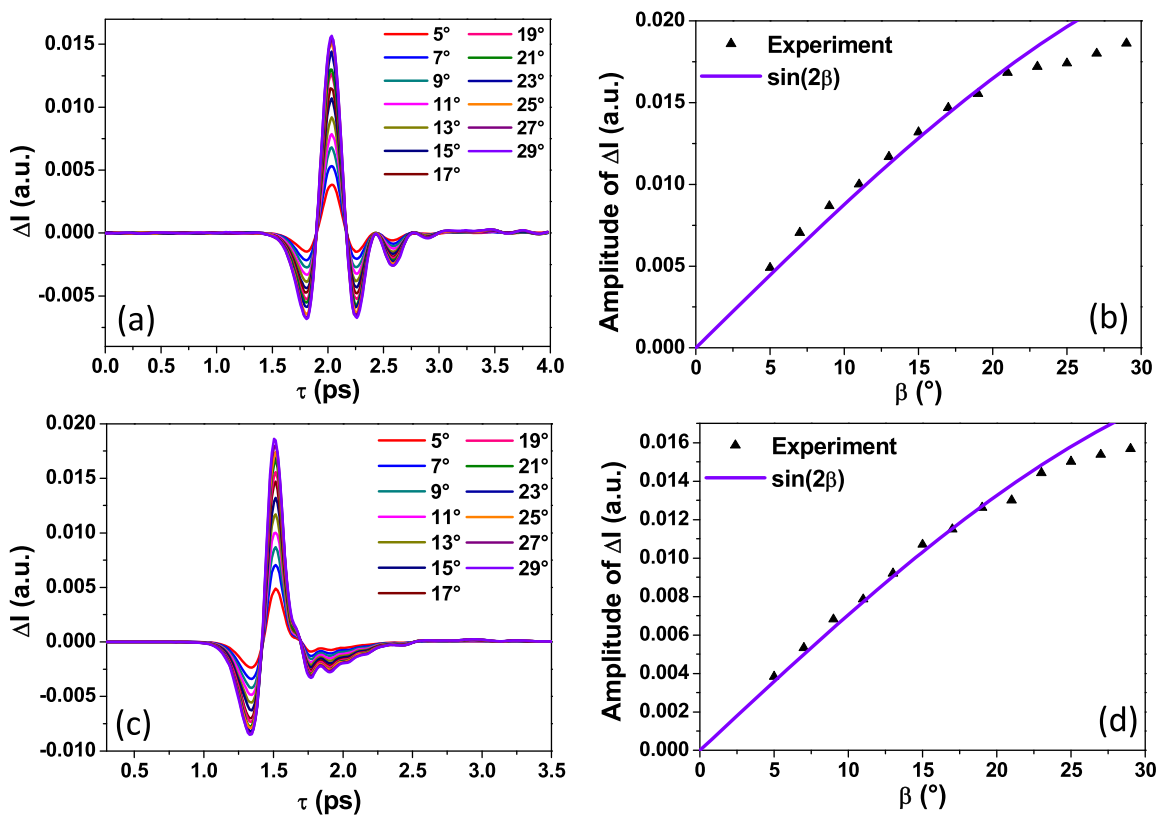

Fig. 3. EO sampling by optical heterodyne detection performed in $\mathrm{ZnTe}[(\mathrm{a})$ and (b)] and GaP [(c) and (d)]. (a),(c) THz electric field, for different $\beta$ angles, versus the time delay $\tau$. (c),(d) Evolution of the amplitude of the signal displayed in (a) and (c) with respect to the heterodyning angle $\beta$. The solid line curve corresponds to a fit of the data with the function $\sin (2 \beta)$.

They share the common characteristic of being pumped in the near infrared, and may require some frequency doubling of the pump pulse for further characterization of the emitted $\mathrm{THz}$ wave by EO sampling [13]. One can avoid the insertion of a nonlinear crystal for $\mathrm{SH}$ generation of the pump beam prior to EO sampling, or the use of a $\mathrm{THz}$ Michelson interferometer to retrieve the $\mathrm{THz}$ waveform by first order autocorrelation [14], by resorting to the heterodyne detection of the $\mathrm{THz}$ wave via $\mathrm{THz}$ field induced second harmonic generation in a plasma [15] or in a suitable EO crystal as we did.

Consequently, since $\mathrm{THz}$ detection through optically heterodyne detected PISH signal deserves some attention, we are going to present it in detail compared to the elementary treatment of [9] where, for instance, no derivation of the ana lytical expression for the measured signal or evaluation of the enhancement factor was given.

\section{A. Experimental Setup and Analytical Expression of the Optically Heterodyne-Detected PISH Signal}

The experimental setup is the same as in [9]. $\mathrm{THz}$ pulses are generated in $\mathrm{LiNbO}_{3}$ by optical rectification of tilted pulse front, $800 \mathrm{~nm}$, femtosecond pulses, and the EO crystal is a $300 \mu \mathrm{m}$ thick $\mathrm{ZnTe}$. A quarter wave plate, with its fast axis oriented at an angle $\pm \beta$ with respect to the $\hat{z}$ axis, is inserted on the optical probe beam pathway in front of the EO crystal (Fig. 4). The $\mathrm{THz}$ beam is modulated by a mechanical chopper. The $\mathrm{THz}$ induced $\mathrm{SH}$ signal, filtered by a $400 \mathrm{~nm}$ bandpass filter and measured by a photomultiplier tube with a monochromator, is recorded by a lock in amplifier as a function of the time delay $\tau$ between the probe and $\mathrm{THz}$ pulses. Note that, contrary to usual heterodyne detection schemes, this method does not require any polarizer prior to the detector.
Without any loss of generality, we model the probe and $\mathrm{THz}$ beams by monochromatic nondepleted waves [9]:

$$
E_{\ell}(t, x)=\frac{1}{2} \mathcal{E}_{\ell}^{\omega}(x) \exp \left[\mathrm{i}\left(\omega t \quad k_{\omega} x\right)\right]+\text { c.c. } \quad(\ell=y, z)
$$

and

$$
\mathbf{E}_{\mathrm{THz}}(t, x)=\frac{1}{2} E_{\mathrm{THz}} \exp \left[\mathrm{i}\left(\Omega t \quad k_{\Omega} x\right)\right] \hat{\mathbf{y}}+\text { c.c., }
$$

where $k_{\omega}=\omega n_{\omega} / c, k_{\Omega}=\Omega n_{\Omega} / c$, and $n_{\omega}$ (resp. $n_{\Omega}$ ) is the re fractive index of the crystal at the probe (resp. THz) frequency. At the entrance of the crystal, for a positive heterodyning angle $+\beta$, the probe beam amplitude writes

$$
\left[\begin{array}{l}
\mathcal{E}_{y}^{\omega}(\beta, 0) \\
\mathcal{E}_{z}^{\omega}(\beta, 0)
\end{array}\right]=E_{0}\left[\begin{array}{cc}
\frac{1+\mathrm{i}}{2} \sin (2 \beta) \\
(1+\mathrm{i}) \sin ^{2} \beta & \mathrm{i}
\end{array}\right] .
$$

The $\langle 110\rangle$ cut ZnTe crystal, due to the applied THz field, is birefringent with the following principal axes [16]: $\hat{\mathbf{e}}_{1}=\hat{\mathbf{x}}$, $\hat{\mathbf{e}}_{2}=(\hat{\mathbf{y}}+\hat{\mathbf{z}}) / \sqrt{ } 2$, and $\hat{\mathbf{e}}_{3}=(\hat{\mathbf{y}}+\hat{\mathbf{z}}) / \sqrt{ } 2$, whose refractive

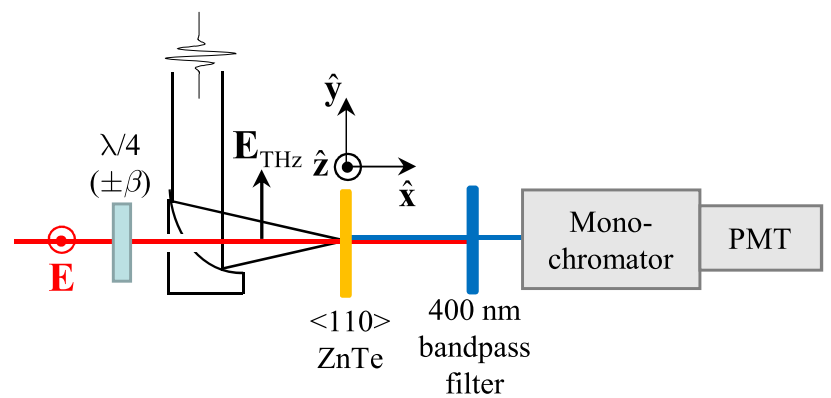

Fig. 4. Experimental setup. PMT, photomultiplier tube; $(\hat{\mathbf{x}}, \hat{\mathbf{y}}, \hat{\mathbf{z}})$, Cartesian frame of the laboratory. 
indices are $n_{1}=n_{\omega}, n_{2}=n_{\omega}+\frac{1}{2} n_{\omega}^{3} r_{41} E_{\mathrm{THz}}$, and $n_{3}=n_{\omega}$ $\frac{1}{2} n_{\omega}^{3} r_{41} E_{\mathrm{THz}}$, respectively. As a consequence, in the frame of the crystal [see Fig. 1(b)], the probe field amplitude at each point $x$ of the crystal is given by

$$
\begin{gathered}
\mathcal{E}_{X}^{\omega}(\beta, x)=\mathcal{E}_{Y}^{\omega}(\beta, x), \\
\mathcal{E}_{Y}^{\omega}(\beta, x)=\frac{E_{0}}{2 \sqrt{ } 2}\left[f_{\beta} \mathrm{e}^{\mathrm{i} \Gamma_{x} / 2}+g_{\beta} \mathrm{e}^{-\mathrm{i} \Gamma_{x} / 2}\right], \\
\mathcal{E}_{Z}^{\omega}(\beta, x)=\frac{E_{0}}{2}\left[f_{\beta} \mathrm{e}^{\mathrm{i} \Gamma_{x} / 2} \quad g_{\beta} \mathrm{e}^{-\mathrm{i} \Gamma_{x} / 2}\right],
\end{gathered}
$$

where

$$
\begin{aligned}
& f_{\beta}=\frac{1+\mathrm{i}}{2} \sin (2 \beta)+(1+\mathrm{i}) \sin ^{2} \beta \quad \mathrm{i}, \\
& g_{\beta}=\frac{1+\mathrm{i}}{2} \sin (2 \beta)+\mathrm{i} \quad(1+\mathrm{i}) \sin ^{2} \beta,
\end{aligned}
$$

and $\Gamma_{x}=2 \pi x n_{\omega}^{3} r_{41} E_{\mathrm{THz}} / \lambda$. These equations make it possible to compute the second order polarization radiating the $\mathrm{THz}$ induced field at frequency $2 \omega$. Its amplitude is given by [17]

$$
\mathcal{P}_{i}^{2 \omega}=\frac{1}{2} \varepsilon_{0} \chi_{i j k}^{(2)} \mathcal{E}_{j}^{\omega} \mathcal{E}_{k}^{\omega},
$$

where $i \neq j \neq k=X, Y, Z$ and $\chi_{X Y Z}^{(2)}=\chi_{Y Z X}^{(2)}=\chi_{Z X Y}^{(2)}=$ $\chi^{(2)}$. In the frame of the laboratory, it writes

$$
\begin{gathered}
\mathcal{P}_{x}^{2 \omega}(\beta, x)=0, \\
\mathcal{P}_{y}^{2 \omega}(\beta, x)=\varepsilon_{0} \chi^{(2)} \frac{E_{0}^{2}}{8}\left[f_{\beta}^{2} \mathrm{e}^{\mathrm{i} \Gamma_{x}} \quad g_{\beta}^{2} \mathrm{e}^{-\mathrm{i} \Gamma_{x}}\right], \\
\mathcal{P}_{z}^{2 \omega}(\beta, x)=\varepsilon_{0} \chi^{(2)} \frac{E_{0}^{2}}{16}\left[f_{\beta} \mathrm{e}^{\mathrm{i} \Gamma_{x} / 2}+g_{\beta} \mathrm{e}^{-\mathrm{i} \Gamma_{x} / 2}\right]^{2},
\end{gathered}
$$

and acts as a source term for the nonlinear wave equation of the SH field amplitude. Within the slowly varying amplitude approximation, this equation is

$$
\left(\frac{\partial}{\partial x}+\alpha_{2 \omega}\right) \mathcal{E}_{\ell}^{2 \omega}=\frac{\mathrm{i} \omega}{c n_{2 \omega} \varepsilon_{0}} \mathcal{P}_{\ell}^{2 \omega} \exp (\mathrm{i} x \Delta k) \quad(\ell=y, z),
$$

where $\alpha_{2 \omega}$ is the absorption coefficient at $2 \omega$ and $\Delta k=$ $2 \omega\left(\begin{array}{ll}n_{\omega} & n_{2 \omega}\end{array}\right) / c$ is the phase mismatch between the fundamen tal and SH fields. Since, in our experiment, $\alpha_{2 \omega} x \gg 1$ and $\alpha_{2 \omega}, \Delta k \gg \Gamma_{x} / x$, one gets, at the output of the crystal:

$$
\begin{aligned}
& \mathcal{E}_{y}^{2 \omega}(\beta, L)=\mathrm{i} \frac{A}{4}\left[f_{\beta}^{2} \mathrm{e}^{\mathrm{i} \Gamma} \quad g_{\beta}^{2} \mathrm{e}^{-\mathrm{i} \Gamma}\right], \\
& \mathcal{E}_{z}^{2 \omega}(\beta, L)=\mathrm{i} \frac{A}{8}\left[f_{\beta} \mathrm{e}^{\mathrm{i} \Gamma / 2}+g_{\beta} \mathrm{e}^{-\mathrm{i} \Gamma / 2}\right]^{2},
\end{aligned}
$$

where $A=\omega \chi^{(2)} E_{0}^{2} \mathrm{e}^{\mathrm{i} \Delta k L} /\left[2 c n_{2 \omega}\left(\mathrm{i} \Delta k+\alpha_{2 \omega}\right)\right]$.

The expansion of $f_{\beta}^{2}, g_{\beta}^{2}$, and $\mathcal{E}_{z}^{2 \omega}(\beta, L)$, assuming that $\Gamma, \beta \ll 1$, makes it possible to calculate the $\mathrm{SH}$ intensity for an heterodyning angle $+\beta, I_{2 \omega}(\Gamma, \beta) \propto\left|\mathcal{E}_{y}^{2 \omega}(\beta, L)\right|^{2}+$ $\left|\mathcal{E}_{z}^{2 \omega}(\beta, L)\right|^{2}$. Neglecting irrelevant terms of order 3 and more in $\Gamma$ and $\beta$, one has

$$
\begin{aligned}
I_{2 \omega}(\Gamma, \beta) \propto & \frac{|A|^{2}}{4}\left[2 \sin ^{2}(2 \beta)+\sin ^{2} \Gamma+\sin (2 \beta) \sin (2 \Gamma)\right. \\
& \left.\frac{3}{2} \sin ^{2}(2 \beta) \sin ^{2} \Gamma\right] .
\end{aligned}
$$

The first term in Eq. (26) corresponds to the intensity of the $\mathrm{SH}$ local oscillator,

$$
I_{\mathrm{LO}}^{2 \omega}=I_{2 \omega}(0, \beta) \propto \frac{|A|^{2}}{2} \sin ^{2}(2 \beta),
$$

whereas the intensity of the $\mathrm{SH}$ homodyne signal, $I_{\text {sig }}^{2 \omega}$, is given by

$$
I_{\text {sig }}^{2 \omega}=I_{2 \omega}(\Gamma, 0) \propto \frac{|A|^{2}}{4} \sin ^{2} \Gamma .
$$

In the terminology of optical heterodyne detection, one has to consider the modulation depth, $M(\Gamma, \beta)=\left[I_{2 \omega}(\Gamma, \beta)\right.$ $\left.I_{\mathrm{LO}}^{2 \omega}\right] / I_{\mathrm{LO}}^{2 \omega}$, of the PISH signal detected for a positive heterodyn ing angle $+\beta$. As $\Gamma, \beta \rightarrow 0$, we found that

$$
M(\Gamma, \beta)=g \Gamma+d(g \Gamma)^{2}+O\left(\Gamma^{3}\right),
$$

with $g=1 / \sin (2 \beta)$ and $d=1 / 2$, results identical to the one found for EO sampling in this context [6].

Finally, the heterodyned PISH signal is

$$
\begin{aligned}
\Delta I_{2 \omega}(\Gamma, \beta) & =I_{2 \omega}(\Gamma, \beta) \quad I_{2 \omega}(\Gamma, \beta), \\
& \propto \frac{|A|^{2}}{2} \sin (2 \beta) \sin (2 \Gamma), \\
& \propto 2 \frac{L}{\lambda} \beta\left[\chi^{(2)} r_{41}\right]^{2} I_{\omega}^{2} E_{\mathrm{THz}} .
\end{aligned}
$$

As expected, the heterodyned PISH signal is proportional to the $\mathrm{THz}$ electric field and makes it possible to measure the latter. Note that, in the equation given in [9] for $\Delta I_{2 \omega}(\Gamma, \beta)$, the square power law dependence regarding $I_{\omega}, \chi^{(2)}$, and $r_{41}$ is missing.

\section{B. Results and Discussion}

Figure 5(a) displays the PISH intensities, $I_{2 \omega}(\Gamma, \beta)$, measured for $\beta= \pm 10^{\circ}$. Both signals oscillate around zero thanks to the mechanical chopper and the lock in amplifier, which make it possible to get rid of the contribution of the local oscillator, $I_{\mathrm{LO}}^{2 \omega}$. Note also that, by adding these two signals, one would get the homodyne signal, $I_{\text {sig }}^{2 \omega}$. On the other hand, their sub straction gives a signal directly proportional to the $\mathrm{THz}$ electric field, as evidenced by Fig. 5 (b) where $\Delta I_{2 \omega}(\Gamma, \beta)=I_{2 \omega}(\Gamma, \beta)$ $I_{2 \omega}(\Gamma, \beta)$ is compared to the $\mathrm{THz}$ waveform measured by CEOS. As can be seen, the agreement between our technique and CEOS is excellent. This is also evidenced by the corre sponding spectra displayed in Fig. 5(c). The signal to noise ratio was found to be $\sim 10^{3}$ in both cases. Note that, contrary to Section 2, these experiments were not performed in dry air, explaining why the measured $\mathrm{THz}$ waveforms and spectra are less clean than those of Fig. 2.

\section{COMPARISON OF THE TWO DETECTION SCHEMES}

First, the two detection schemes presented above offer the same performances in terms of enhancement factor and distortion parameter. So, the choice of one technique rather than the other will depend on the user's aim. If one intends to measure $\mathrm{THz}$ pulses generated by amplified Ti:sapphire laser systems, the recommended scheme is the optically heterodyne detected electro optic sampling (Section 2) for the following reasons: first of all, this setup is very close to the widespread CEOS setup 

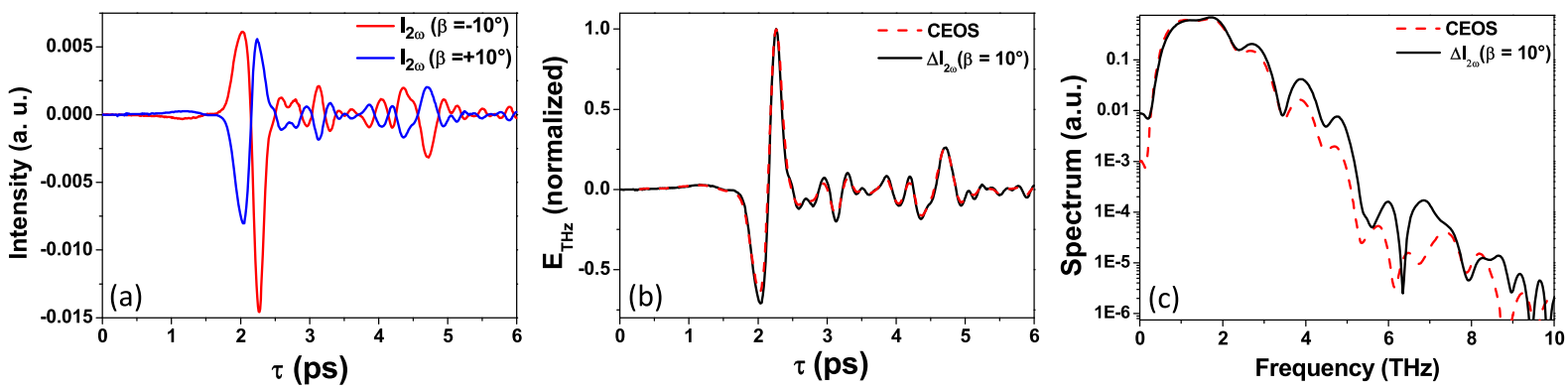

Fig. 5. (a) PISH signals for two opposite heterodyning angles: $\beta=10^{\circ}$ and $\beta=10^{\circ}$. (b) EO signal measured with a CEOS setup (dashed red curve) and heterodyned PISH signal difference, $\Delta I_{2 \omega}$, for $\beta=10^{\circ}$ (black curve). All the signals are plotted as a function of the time delay, $\tau$, between the probe and $\mathrm{THz}$ pulses. (c) Corresponding spectra.

usually dedicated to this task; it makes it possible to acquire the $\mathrm{THz}$ waveforms in one scan, limiting the distortions that may arise from any drift of the experimental setup between two consecutive scans for two different heterodyning angles; finally, it does not require the use of a photomultiplier tube, which is much more expensive and fragile than a photodiode. However, this scheme, like the second one, requires good polarizing optics (polarizers, wave plates). Furthermore, these optics must be identical (i.e., from the same manufacturer) on both arms after the EO crystal in order to reduce as much as possible the influence of any intrinsic birefringence. As already mentioned in Section 3, the second detection scheme is interesting if one intends to characterize $\mathrm{THz}$ pulses from a setup pumped by near infrared laser pulses. It is a good alternative to much more complex detection techniques, like $\mathrm{THz}$ field induced second harmonic generation in a plasma or the use of a Michelson interferometer, since it is very close to the setup of CEOS and requires very few modifications of the latter to be imple mented in the laboratory.

\section{CONCLUSION}

Two simple and distortion free methods to sample $\mathrm{THz}$ pulses in zinc blende type crystals, based on an optical heterodyne detection scheme, have been presented and demonstrated experimentally. The first one makes it possible to measure, in a single measurement and without distortion, a THz waveform by detecting an optical probe beam. Regarding the second one, its relies on the so called PISH effect and makes it possible to sample a $\mathrm{THz}$ wave through $\mathrm{THz}$ induced second harmonic generation. It might be useful in characterizing $\mathrm{THz}$ pulses generated by optical rectification of near infrared pulsed sources other than Ti:sapphire lasers. Both methods are as good as the more conven tional, and widespread, electro optic sampling technique.

Funding. Conseil Régional d'Aquitaine (2011 1603 004).

Acknowledgment. M. C. is grateful for thesis funding from the Direction Générale de l'Armement (DGA) and the Région Aquitaine.

\section{REFERENCES}

1. Q. Wu and X. C. Zhang, "Ultrafast electro optical field sensors," Appl. Phys. Lett. 68, 1604-1606 (1996).
2. L. Duvillaret, S. Rialland, and J. L. Coutaz, "Electro optic sensors for electric field measurements. II. Choice of the crystals and complete optimization of their orientation," J. Opt. Soc. Am. B 19, 2704-2715 (2002).

3. N. C. J. van der Valk, T. Wecnkebach, and P. C. M. Planken, "Full mathematical description of electro optic detection in optically isotropic crystals," J. Opt. Soc. Am. B 21, 622-631 (2004).

4. Z. P. Jiang, F. G. Sun, Q. Chen, and X. C. Zhang, "Electro optic sampling near zero optical transmission point," Appl. Phys. Lett. 74, 1191-1193 (1999).

5. A. Schneider and P. Günter, "Measurement of the terahertz induced phase shift in electro optic sampling for an arbitrary biasing phase," Appl. Opt. 45, 6598-6601 (2006).

6. F. D. J. Brunner, J. A. Johnson, S. Grübel, A. Ferrer, S. L. Johnson, and T. Feurer, "Distortion free enhancement of terahertz signals measured by electrooptic sampling. I. Theory," J. Opt. Soc. Am. B 31, 904-910 (2014).

7. J. A. Johnson, F. D. J. Brunner, S. Grübel, A. Ferrer, S. L. Johnson, and T. Feurer, "Distortion free enhancement of terahertz signals measured by electrooptic sampling. II. Experiment," J. Opt. Soc. Am. B 31, 1035-1040 (2014).

8. M. Cornet, J. Degert, E. Abraham, and E. Freysz, "Frequency resolved cross correlation between optical and terahertz pulses: application to ultrashort laser pulse characterization," Opt. Express 24, 3003-3010 (2016).

9. M. Cornet, J. Degert, E. Abraham, and E. Freysz, "Terahertz field induced second harmonic generation through Pockels effect in zinc telluride crystal," Opt. Lett. 39, 5921-5924 (2014).

10. M. D. Thomson, M. Kreß, T. Löffler, and H. G. Roskos, "Broadband $\mathrm{THz}$ emission from gas plasmas induced by femtosecond optical pulses: from fundamentals to applications," Laser Photon. Rev. 1, 349-368 (2007).

11. G. R. Fowles, Introduction to Modern Optics (Dover, 1989).

12. K. L. Vodopyanov, J. E. Schaar, P. S. Kuo, M. M. Fejer, X. Yu, J. S. Harris, V. G. Kozlov, D. Bliss, and C. Lynch, "Terahertz wave gener ation in orientation patterned GaAs using resonantly enhanced scheme," Proc. SPIE 6455, 645509 (2007).

13. Z. Yang, L. Mutter, M. Stillhart, B. Ruiz, S. Aravazhi, M. Jazbinsek, A. Schneider, V. Gramlich, and P. Günter, "Large size bulk and thin film stilbazolium salt single crystals for nonlinear optics and $\mathrm{THz}$ genera tion," Adv. Funct. Mater. 17, 2018-2023 (2007).

14. C. Vicario, M. Jazbinsek, A. V. Ovchinnikov, O. V. Chefonov, S. I. Ashitkov, M. B. Agranat, and C. P. Hauri, "High efficiency $\mathrm{THz}$ gen eration in DSTMS, DAST and $\mathrm{OH} 1$ pumped by Cr:forsterite laser," Opt. Express 23, 4573-4580 (2015).

15. N. Karpowicz, J. Dai, X. Lu, Y. Chen, M. Yamaguchi, H. Zhao, X. C. Zhang, L. Zhang, C. Zhang, M. Price Gallagher, C. Fletcher, O. Mamer, A. Lesimple, and K. Johnson, "Coherent heterodyne time domain spectrometry covering the entire "terahertz gap"," Appl. Phys. Lett. 92, 011131 (2008).

16. A. Yariv, Quantum Electronics (Wiley, 1989).

17. P. N. Butcher and D. Cotter, The Elements of Nonlinear Optics (Cambridge University, 1990). 